\title{
DEVELOPMENT OF AN INTEGRATED TOOL THAT SUMMARRIZE AND PRODUCE THE SIGN LANGUAGE FOR WEB PAGES TO PROMOTE WEB ACCESSIBILITY FOR DEAF
}

\author{
Ahmad A. Alhamed ${ }^{1}$ and Maha s. Alqhtani ${ }^{2}$ \\ ${ }^{1}$ Department of Information Systems, King Saud University, Riyadh, KSA
}

\begin{abstract}
This paper presents an intelligent tool to summarize Arabic articles and translate the summary to Arabic sign language based on avatar technology. This tool was designed for improving web accessibility and help deaf people for acquiring more benefits. Of the most important problems in this task, is that the deaf people were facing many difficulties in reading online articles because of their limited reading skills. To solve this problem the proposed tool includes a summarizer that summaries the articles, so deaf people will read the summary instead of reading the whole article.Also the tool use avatar technology to translate the summary to sing language.
\end{abstract}

\section{KEYWORDS}

Accessibility, Arabic sign language, Key terms summarizer, Avatar.

\section{INTRODUCTION}

The world wide web has evolved very fast in recent years and become necessary for everybody. It is a rich source of information and documents in all fields. Unfortunately, deaf people cannot take full advantage of these advances in technology. Some Web sites are not sufficiently accessible to deaf people, making it difficult or impossible for them to contribute to the Web.

Web accessibility means that the web is accessible to every body even to people with disabilities. In another word, people with disabilities can perceive, understand, and interact with the Web . There are many attempts to improve the web accessibility by providing closed captioning and transcriptions, whenever a sound appears. In addition, the accessibility for deaf can be improved with the utilization of the sign language.

Deaf people face problems in reading electronic documents or articles that are available on certain web pages. They have a hard time to understand the written language because of their limited vocabulary and language skills. The poor language capability for deaf people is because they cannot listen and practice the basic language techniques[46]. According to the previous studies in the field of the deaf, reading level of the deaf is lower than the reading level of hearing people. Furthermore, the level of experience achieved by deaf children in four years is equivalent to the level of one year for hearing children [24]. 
Most web pages offer written information such as news, articles or other documents. These web pages may lose a number of visitors who are deaf. Because deaf readers have difficulties with many skills that are required for reading such as word identification, vocabulary .Also they have problems with reader-based skills like working memory [16].With increased advancement of technology text summarization has a number of applications that are used in the analysis of a high volume text documents. With text summarization tools the online documents can be summarized into a shorter form .So, deaf can read only the summary instead of reading the whole document. Automatic text summarization tools can be helpful for deaf people by reducing the time and efforts needed to read and understand the documents . Moreover, many deaf people follow sign language which is a lot easier than the written word. Web accessibility can be improved be integrating text summarization tools with sign language to provide better access for the deaf.

This paper aims to develop a new tool to summarize the online articles in order to produce a summary that contains the main points of the original articles. And interpretation of these summarized articles to sign language by using 3D avatar technology.

The proposed tool will be responsible for two major tasks. The first task is to build Automatic Arabic text summarization tool to summarize certain Arabic articles to create shorter versions of these articles. There are two types of summarization extractive and abstractive. Extractive by selecting important pieces from the original documents to produce shorter form. An Abstractive summarization generates summaries whose material is not presented in the input text by understanding of the main concepts in a document and then use new words that are not present in the original document to express these concepts. Abstraction methods are still difficult to achieve because it requires text analysis, modelling, and language generation techniques [41].

There are different methods to implement extractive summaries. The most important ones are: the linear methods that give a score for each sentence depending on heuristic measures, such as Naive Bayesian classifier ,Latent Semantic Analysis (LSA) which is inspired by latent semantic indexing .And a Graph Based methods that represent the sentences in the text as nodes with a directed graph; the two sentences are connected if its share some words may its refer to some similarity [41].The second task of the proposed tool is to translate the output summary form the previous step to Arabic sign language using avatars technology. Deaf people use sign language as their first language to communicate with others[10]. Sign language relies on facial expressions and body language to convey meaning, rather than transferred to the acoustically sound patterns.

The proposed tool will include a dictionary of the most used words in Arabic with their corresponding sign files. Visualization of signs by using computers have been evolved from pictures to video clips and finally to 3D Avatar. We use 3D Avatar because sign files are very smaller than videos and pictures; thus the amount of storage space required is minimal. Also, the possibility of moving the 3D avatar at different angles making it easy for the deaf to understand the signal[8].

The main objective of this paper is to improve web accessibility for deaf people by developing a tool facilitating communication through the Web for deaf. This tool will summarize Arabic articles and translate the output summary to sign language. Thus will help deaf people to convey the meaning of these articles in less time and efforts. 


\section{RELATED WORK}

This part presents a review of previous studies related to web accessibility for deaf people, automatic Text summarization and translating Arabic Text to ArSL:

\subsection{Web Accessibility:}

The accessible websites enable people with disabilities to do many things such as reading and online shopping without having to rely on others. He proposes a model and a set of guidelines to bring awareness of accessibility on the Web. These guidelines help developers to developed accessible websites. If a website does not take into account the needs of disabled people then, these people can be suffer from using such sites because of inaccessible design[25]

An attempt in [28] for making e-learning available to persons with disabilities. It presented a Web accessibility framework for the Arab disabled users and facilitated their learning. The proposed system depends on extracting the website content via reading; then the derived content is presented in ArSL animations for the deaf users or speech for the blind users. In order to translate the extracted material the system use ArSL animated library which consists of each Arabic word and the corresponding ArSL animated GIFs. This system implemented in PHP language and MySQL. Improving web accessibility for the deaf people is relatively possible and inexpensive. The best solution is the use of closed captioning and transcriptions, whenever a sound appears. Web accessibility can also be improved with the utilization of the sign language. Most people who are deaf understand and use the sign language which is easier than the written word. Unfortunately, implementation of sign language is expensive because it requires a sign interpreter. In order to help the deaf user to understand the audio material. Some websites involve captioning within the video or provide a link located near the video or audio material to access the captions and transcriptions .

Improving web accessibility for the deaf people is relatively possible and inexpensive. The best solution is the use of closed captioning and transcriptions, whenever a sound appears. Web accessibility can also be improved with the utilization of the sign language. Most people who are deaf understand and use the sign language which is easier than the written word. Unfortunately, implementation of sign language is expensive because it requires a sign interpreter. In order to help the deaf user to understand the audio material. Some websites involve captioning within the video or provide a link located near the video or audio material to access the captions and transcriptions .

\subsection{Automatic Text summarization:}

R. Dixit and S.Apte [39] proposed a method for text summarization based on fuzzy logic to extract important sentences based on their features as a summary. The fuzzy logic system includes four components: fuzzifier, defuzzifier , an inference engine, and the fuzzy knowledge base. In the fuzzifer, inputs are translated into linguistic values by using a membership function. Then, the inference engine uses fuzzy IF-THEN rules to derive the linguistic values. In the final step, the output variables from the inference engine are converted to the final values by the defuzzfier using a membership function for representing the final sentence score. Then all the sentences of a text are ranked in a descending order according to their scores. Sentences that have the highest score are extracted as a text summary.

G. Al_Gaphari et al.[16] presented and discussed a graph based centrality algorithm for automatic Arabic text summarization. The graph-based algorithm extracts the most important sentences in a 
document by computing the similarity between two sentences and assessing the centrality of each sentence in a cluster. The researchers use a real-world dataset for testing and validating the software summarizer performance, and the result was very promising. They concluded that the proposed summarizer may outperforms other summarizers used for text summarization. Sobh et al. [40] Integrates Naive Bayesian and Genetic Programming (GP) classification methods in an optimized way to extract the summary. The process of automatic summarization is becoming very important with an increased number of information sources available on the web. The proposed system use Naive Bayesian classifier and Genetic Programming classifier in parallel. Naive Bayesian Classifier classifies each sentence based on its features such as Sentence length, position in the paragraph, or Sentence similarity. Genetic Programming Classifier is automated learning of computer programs. GP was used in many fields, for example, financial market, image processing, signal processing, and pattern recognition. Authors choose to use the Discipulus1 GP system which is the world's first and fastest Genetic Programming system that writes computer programs automatically in Java, C, and Intel assembler code. They used the downloadable free version with default settings for cross-over when running the tool for classification. Authors concluded that by integrating both classifiers, the union for integration increases the result summary and intersection for integration decreases the summary size. Finally, the proposed system is optimized and able to produce summaries comparable to human-generated summaries.

Many systems have been investigated in the area of automatic text summarization, and some of these are available online, and others are used as commercial software. In this section, we will list some of these systems

- SweSum: It is automatic text summarizer for Swedish by Martin Hassel and Hercules Dalianis. It summarizes Swedish news in HTML/text format on the web. This system calculates the frequency of the keywords, in which sentences they appeared, based on statistical and heuristic methods .

- WebSummarizer: It is an application to summarize web pages and documents to produce interactive outlines and Visual Summaries. It also offers summarization of Wikipedia articles and supports many languages such as German ,French and Spanish

- TextCompactor: This summarizer calculates the frequency of each word in the text. Then, each sentence score is calculated based on the frequency count of the words it contains. The most important sentence is considered to be a sentence with the highest frequency count.

\subsubsection{Arabic Text Summarization Systems:}

- The Summarizer of AramediA: This Summarizer used for summarizing Arabic and English texts. It extracts the important sentences based on linguistic analysis of the input text. The user can preview these ideas instead of reading the whole document .

- Sakhr Summarizer: Sakhr is a commercial online Arabic text summarization system. This Summarizer identifies the most relevant sentences within a text based on a set of textmining tools. The Summarization engine applies the Sakhr Corrector to correct the input Arabic text automatically from the common Arabic errors, and the Keywords Extractor to identify a list of important keywords that to identify the important sentences [41].

- SARA@ : Is an automatic Arabic text summarization developed by RDI ( Research \& Development International). It extracts the most important sentences based on the morphological analysis of the words and mathematical analysis of the sentences and their 
relationships. The user of the system can easily control the size of the summary (e.g., $20 \%$ or $50 \%$, and so on).And the system is valid for all types of texts such as news, science, arts, sports, etc.

\subsection{Text to Sign Language}

Wraya, et. al, [4] presented a system designed to support transactions between a post office clerk and a deaf customer. This interactive translates the clerk's speech into British Sign Language (BSL) and displays the signs on the screen, using a specially-developed avatar. However, the system is limited to post office operations. The system operates through a series of stages, start when a deaf person enters the post office and requests the service using gestures. Once the clerk know that the customer is deaf he/she start speaks into his/her headset microphone. Then the automatic speech recognizer selects possible messages from an extensive set of pre-entered targets and displays those with the highest probability on a screen. The clerk confirms the message intended. Then the message contents, are translated into a set of sign instructions and displayed on a video screen. The authors recognized that the system potentially offers access to a range of information that they may previously have had difficulty obtaining.. The developed system achieved an accuracy of identification of the signed phrases of $61 \%$ for complete phrases and $81 \%$ for sign units. Email is a convenient communication tool for most of the people. Unfortunately, deaf people cannot take the full advantages of these techniques. Non-deaf people are easy to use Email to communicate with their family, friends or other people. However, deaf people have difficulties to study the nature language. They have a hard time to write a proper sentence or even a short mail because they have limited vocabulary capabilities and weak language skills. When they read a sentence, they do not understand the whole sentence properly. Authors proposed a method to convert Chinese texts to a speech and sign language simultaneously. With this proposed system, a deaf people can easily read an Email or articles by a translated sign language stream [47]. M. JEMNI and O. ELGHOUL, [27] Developed a Webbased interpreter of Sign Language (SL). This tool facilitates the communication between hearing people who do not know sign language and deaf individuals. Authors discussed three main approaches: - Writing based systems The first transcription of signs languages based on writing or drawing symbols. Many systems of transcription appeared like HamNoSys and SignWriting. These systems used to encode sign language in a linear way.

\subsubsection{Video-based Systems}

The video-based systems are a video sequence corresponding to a human interpreter. This video sequence has many limitations in performance, the contents are not easily reusable, and therefore a human translation in a language of signs is necessary and remains very costly [27].

\subsubsection{Avatar based Systems}

Avatar based systems this new technological context, involve the creation of a virtual character. Avatar is more precisely and more interested in communication systems in sign language by animation of virtual 3D persons. The proposed tool is called WebSign it based on the technology of avatar. The input of the tool is a text in natural language, and the output is a real-time interpretation in sign language. The tool uses a dictionary of word and signs to perform the interpretation. The first version of Web Sign tool is finalized, and the authors attempt to contacts with specialized deaf associations to train users and evaluate the system [27].

M. Mohandes [29] discussed the importance of Sign Language for deaf people as their natural way of communication just like a speech for non-deaf people. Deaf people often communicate with non-deaf people through a sign language interpreter. Sign language interpreter is not always available when needed. Therefore, there is an increasing need to provide an automatic interpreter for translating signs to speech and the reverse using the modern technology. He proposed a 
system that converts Arabic text to Arabic sign language. The system was developed using Java Server Pages (JSP), implicitly having all the benefits of the Java programming language. The system has a database to store Arabic words with the corresponding signs representation. When the word is typed a letter by letter, the system automatically starts searching for the word if the word is available in the database then the recorded clip will be shown. If the word does not have a corresponding sign in the dictionary, then finger spelling is done by displaying images that representing each letter of the word. The author concluded that this proposed system can be used to teach deaf or their families the Arabic Sign Language.

\section{Web AcCessibility and Deaf People}

Deafness has different meanings, but those meanings are relevant in a way or another; however, different definitions of deafness have been given; the Wikipedia, defined deafness as following:

"Hearing loss, deafness, hard of hearing, or hearing impairment is a partial or total inability to hear. It affects the development of language for children and for adults it can cause work-related difficulties".

Deaf people are people who suffer from a weakness or loss of hearing. Deaf people are facing many difficulties when communicating with their society. The main solution to help deaf people is the use of Sign language. Sing language provides an easy way of communication with each other's and it is the easiest way to learn for any deaf. When they want to interact with normal people, they need a translator who understands both languages.

Deaf people use the internet, like any other non-deaf people. While the Web provides a large amount of information and resources in all fields of knowledge, it also poses difficulties when content is not designed to be accessible. Deaf users cannot take full advantage of the web because of their hearing disability. Because they are deaf they cannot listen to any video; they cannot listen to any audio. For example, if deaf users want to use YouTube they cannot listen to any of video of audio material which causes boringness in the film. Deaf people cannot take full advantage of the web they cannot even listen to any educational video to increase their knowledge. Some users with a minor hearing disability can be treated and helped by using some mechanical instruments to get them hear full sound. But such devices are expensive. Another difficulty is that many deaf people do not have highly developed reading skills. Because they have a limited reading vocabulary. So, the access to the information on web pages will be difficult.

Some web pages are designed to be accessible for all users even for people with disabilities by providing content suitable for all type of users this is called web accessibility. Web accessibility can be defined as that people with disabilities can use the Web, i.e., can perceive, understand, and interact with the Web. When web pages are accurately designed or made, developed and modified then all users or clients have equal access to information and functions.

Most Web sites have accessibility obstacles that make it difficult for many people with disabilities to use the Web sites. Here some types of web accessibility obstacles that deaf people commonly encounter from poorly designed websites and web tools .

- Lack of sign language to supplement the information and text that is difficult to read.

- Web sites that offer Audio content, such as videos with voices, without captions or transcripts. 
- Web sites that include captions or transcripts for the Audio or video contents but do not present options to adjust the text size and colors for captions.

- Web sites that offer services, including web applications, which rely on interaction using voice only.

\section{Arabic Sign Language}

Sign Language (SL) is an essential communication tool used among deaf people. Sign language is non-voice communication used by people with special needs impaired (deaf) or audio (dumb). Sign language relies on facial expressions and body language to convey meaning, rather than transferred to the acoustically sound patterns [5]. Sign language primary components consist of Manual Features (MF) and Non-Manual Features (NMFs) .Manual Features are signs that are presented by one or both hands in different shapes,movements, orientations and locations. While NMFs are features that do not require hands, it consist of the several facial expressions, head tilting, mouthing, shoulder raising, , and similar signals that we add to our hand signs to create meaning, feeling and/or represent the morphological and syntactic markers of a sentence [5].

As an independent language, Arabic sign language has its own rules. ArSL is limited to represent nouns, verbs, and adjectives. Its sentences have particular characteristics can be illustrated as follow:

- Names: There is no exact representation of the personal name in sign language. Instead, deaf people depend on a special mark of a person to describe that person such as a big nose, his job ...etc. Also, finger spelling is used to spell out the name of a person or any nouns .

- Verbs: are used in a simple form without specifying the time (past, present, future). Verbs are attached with words that indicate the time of occurrence (tomorrow, yesterday, now ...etc) .

- Adjectives: This is one of the most important parts of any sentence. It is used in the Arabic language to simplify the structure of the sentences.

- Plural: are represented as a singular attached with the sign of number that indicates the quantity .For example, the word (كتابان) are expressed in sign language by two signs one for the word (كتاب) and the other for the sign of the number two .

- Prepositions and adverbs: are expressed in the context of articulation by specifying movements, orientations, and locations [5].

\subsection{Importance of Sign Language}

Sign Language (SL) is an essential communication tool used among deaf people. Sign language is non-voice communication used by people with special needs impaired (deaf) or audio (dumb) . Sign language relies on facial expressions and body language to convey meaning, rather than transferred to the acoustically sound patterns. Sign language primary components consist of Manual Features (MF) and Non-Manual Features (NMFs) .Manual Features are signs that are presented by one or both hands in different shapes, ,movements, orientations and locations. While NMFs are features that do not require hands, it consist of the several facial expressions, head tilting, mouthing, shoulder raising, , and similar signals that we add to our hand signs to create meaning, feeling and/or represent the morphological and syntactic markers of a sentence The importance of sign language rise with the need for a way of interaction between deaf people and to communication with their society. It helps deaf to express themselves, their needs and interests. It also developed their skills and experiences. Since it is their natural language, sign 
language can be understood than any other ways of communication. Sign language now imposed itself as an official language and has become essential for deaf students, and those who deal and communicate with the deaf [5].

\subsection{Avatar based Technology VS Video Files}

The video based systems consist in the insertion of a video sequence of a human interpreter in the original video tape. Video sequence coding into sing language has some limitations; such as the contents cannot be used many times and therefore a human sign language interpreter is necessary [27].

Methods for visualization of signs by using computers have been started called Avatar which is currently the best and have a lot of features. The Following are the main points of difference between the 3D avatar and video files Table 1[45].

Table 1 The main differences between 3D avatar and video files.

\begin{tabular}{|l|l|}
\hline \multicolumn{1}{|c|}{ Avatar } & \multicolumn{1}{|c|}{ Video Files } \\
\hline $\begin{array}{l}\text { Avatar animation systems can be easily } \\
\text { controlled and customized by users at a low } \\
\text { cost. }\end{array}$ & $\begin{array}{l}\text { The content of the video cannot be modified } \\
\text { after production. It depends on the sign } \\
\text { interpreter who carried out and the video } \\
\text { sequence is limited to the original positioning of } \\
\text { the camera . }\end{array}$ \\
\hline $\begin{array}{l}\text { It can be viewed from several points of } \\
\text { reference, and it helps in learning the signal } \\
\text { correctly. }\end{array}$ & A fixed point of view and cannot be changed. \\
\hline $\begin{array}{l}\text { Sign files are very small .Thus the amount of } \\
\text { storage space required is minimal. }\end{array}$ & Signal file size is too large. \\
\hline $\begin{array}{l}\text { It can be downloaded on the Web pages } \\
\text { quickly because of their small size. }\end{array}$ & $\begin{array}{l}\text { It may take some time to load if the internet } \\
\text { speed is slow. }\end{array}$ \\
\hline $\begin{array}{l}\text { Provides a low-cost means for adding sign } \\
\text { language translation to any media because } \\
\text { animation is much easier type of data than } \\
\text { video to store. }\end{array}$ & $\begin{array}{l}\text { Recording a signer with a video camera, it seems } \\
\text { to be costly. }\end{array}$ \\
\hline
\end{tabular}

\section{TOOL DESIGN AND IMPLEMENTATION}

In this section, we describe the design of our tool that summarize Arabic text and translate it to Arabic sign language.

\subsection{Tool Design}

The proposed tool architecture is illustrated in Figure 1.The tool is divided into two main phases, the key terms summarizer and the process of converting the output summary to sign language. 
International Journal of Information Technology, Control and Automation (IJITCA) Vol.7, No.3, July 2017

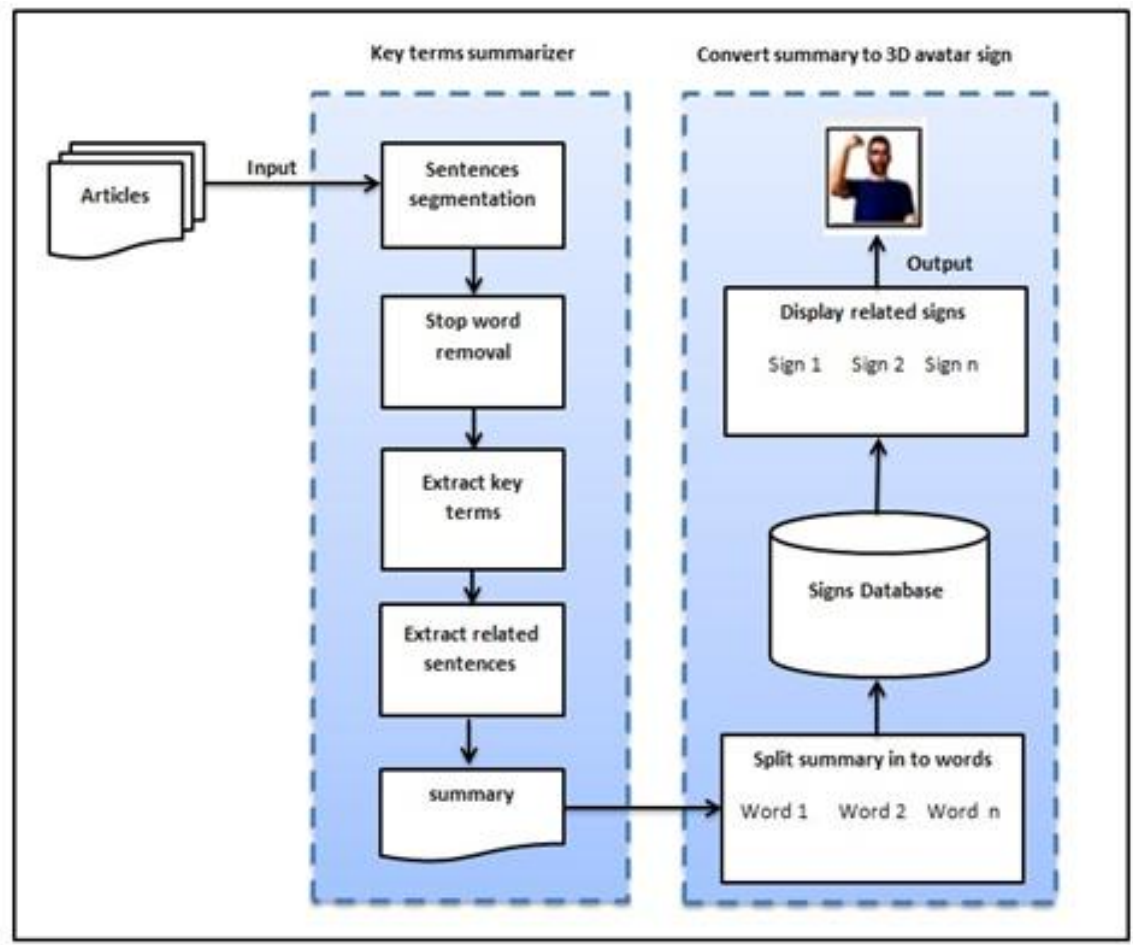

Figure 1: Proposed Tool Architecture

The process goes into several steps:

- Key Terms summarizes read the text and then identify the boundaries of the sentences.

- Remove stop words and extract the Key terms which is the most frequent terms in the article.

- Extract the summary by extracting only the shortest sentence between the sentences belonging to the same key term.

- Convert the extracted summary into sign language by phrasing the summary into words.

- Search the signs data base for the related signs.

- Display the signs in the web page as animation by the signing avatar.

\subsection{Tool Implementation}

The tool was implemented using PHP language to integrate the tool components. In this subsection we will talk about our tool development phases: 1) summarizer building; 2) signs building; 3) Database (DB) building; 3) user interface design.

\subsubsection{Summarizer building}

\subsubsection{Read TXT File}

In this phase we will use PHP programming language to read an Arabic file into our tool,and then we will apply our approach on it. 


\subsubsection{Sentence Segmentation}

Segmentation is the process of identifying the boundaries of Segmentation is the process of determining the boundaries of the sentences. In Arabic language punctuation marks are used to divide the sentences which determine the end of each sentence, such as question marks

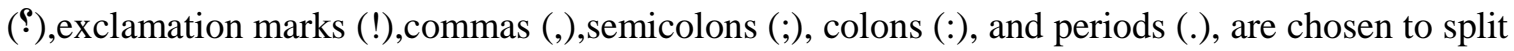
the text into sentences.

\subsubsection{Stop Word Removal}

This algorithm will remove the Arabic stop words which consist of the following word categories: Adverbs, Prepositions, Pronouns, Conditional Pronouns, Interrogative Pronouns, Referral Names/ Determiners, Relative Pronouns, Transformers (verbs, letters), Verbal Pronouns .

\subsubsection{Key terms extraction}

The algorithm will extract the Key term which is the most frequent term in the text, by scanning the text for the terms and calculate the key terms frequency in the original text by applying this equation:

Key term rank $=\Sigma$ (Key term Frequency)

Only one sentence of the sentences that are belonging to one Key term will be extracted .In order to avoid redundant selection of the sentences the length for each sentence will be calculated and give a rank for each sentence, then extract only the shortest sentence between the sentences belonging to the same Key term to be in the output summary. The length of sentence will be calculated using this formula :

Length of Sentence $=\Sigma($ Count Of All Terms In Sentence $)$

\subsubsection{Algorithm}

For our tool we will use the following algorithm:

1. Load text file

2. Make pre-processing phases:

2.1 Sentences segmentation

2.2 Stop word removal

3. Extract and rank Key terms

(The most frequent terms in the text)

4. Extract the summary

(The sentences which are referring to one Key terms)

If (two sentences belong to the same Key term)

- Calculate the length of every sentence

(Number of terms in the sentence)

- Extract the sentence with the shortest length

5- Display the summary.

\subsubsection{Signs Building}

For the representation of signs we used a 3D animated virtual agent, the avatar (VGuido) developed by Televirtual Ltd. Also we used HamNoSys to generate the animations from the descriptions of signs. HamNoSys is a system for writing signed languages was developed by 
Hamburg University.Since computers cannot process the symbols of HamNoSys, SiGML, was used to translate HamNoSys symbols into SiGML which is a modified version of XML.

This information is processed in a synthesizer, which, taking into account the description of the geometry of the animated agent. Finally, the description of the signs is represented by the virtual avatar .

Also we use eSign software which is an Editor tool, designed in the project eSign provides an editing environment .In eSign editor document window, the signed text is represented by sequences of glosses. Glosses are the labels for signs. Frist, we select one technical article and after summarizing it using previous step. Then we synthesis the words that are presented in that summary by achieving the following steps:

1- We created a gloss for each word and then from HamNoSys window we enter the notations of manual and non-manual movements as needed (Figure 2).

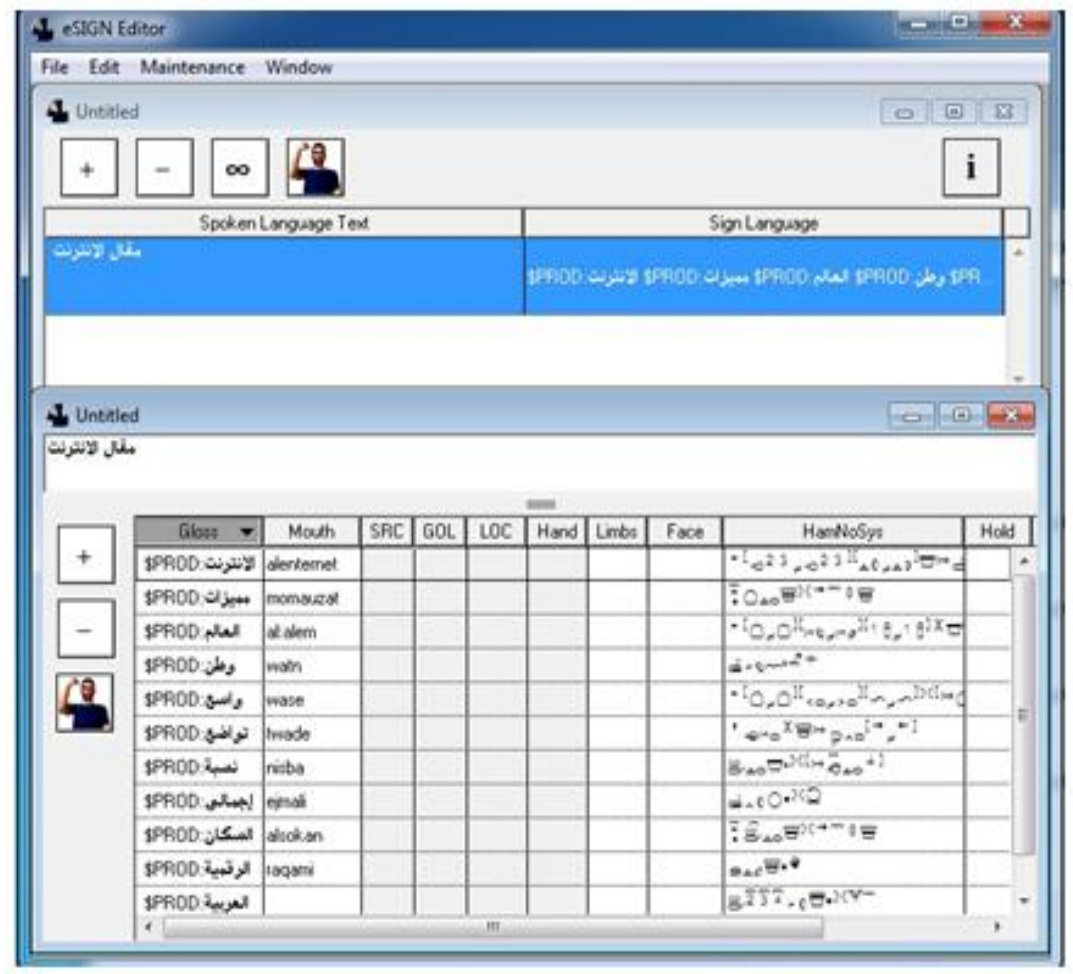

Figure 2 Forming Glosses and HamNoSys in eSign editor.

2- Saving the list of closes as a SiGML file.

3- To play any sign by avatar character we select any glosses and press the button of avatar. which is another aspect of eSign editor is that the users can preview the signs before saving it to do any needed modification. For example we play the sign of word internet in Arabic.

\subsubsection{Database (DB) building}

We used My SQL Server to build a small DB consisting of single table called words, which holds the word IDs and signs files. 


\subsubsection{User Interface}

We developed a simple Arabic user interface Figure 3 using PHP language that allows users to read an Arabic article then press the summarization button to show the result of the summarization and translation of the summary process as a set of signs in the output panel.

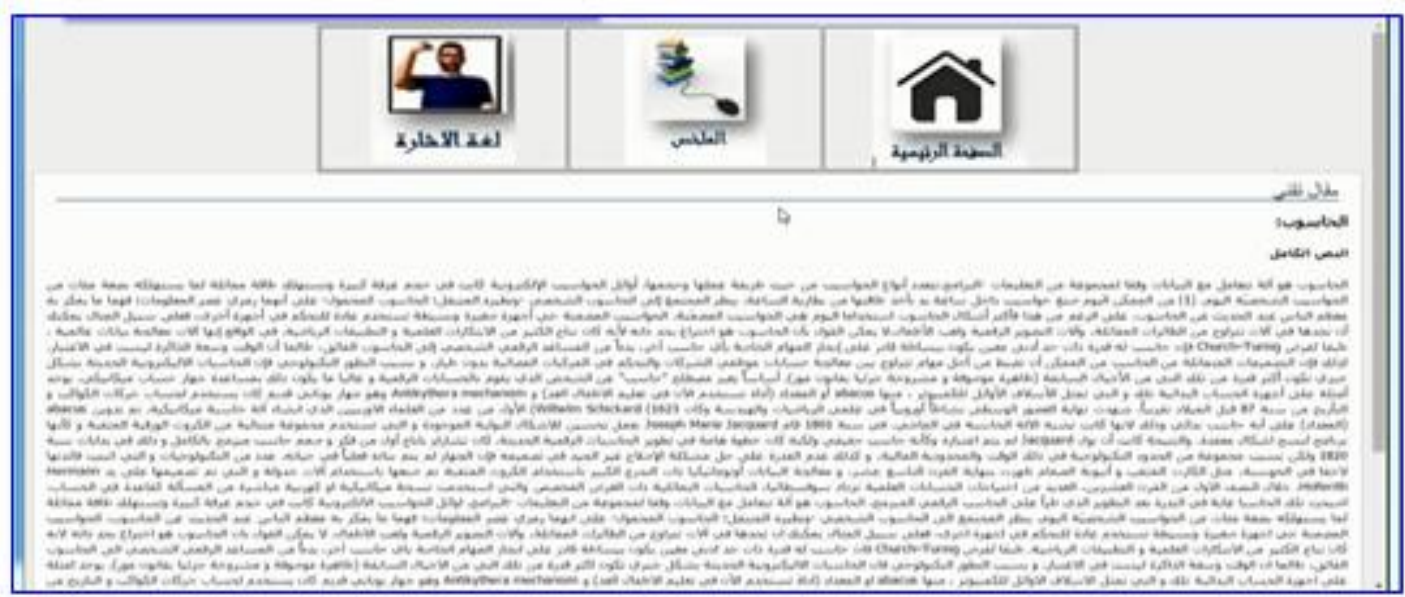

Figure 3: The Proposed Tool Interface

\section{Conclusions}

In this paper, a tool to help deaf people to get better access to the web was proposed. The proposed tool is composed of two main phases; summarizing and translating phase. For summarizing phase we select key terms summarizer which produce a summary .This summarizers show promising result .Second phase include the creating of Arabic signs using 3D avatar technology and store these signs in the database. Deaf people have difficulties in reading skills for that reasons our tool provide a simple user interface with clear icons. Moreover, additional improvements can be applied for this tool in the future upon the needs of deaf people.

\section{ACKNOWLEDGEMENTS}

The authors would like to thank all people who contributed in make this accomplishment.

\section{REFERENCES}

[1] Aarti Patil, Komal Pharande, Dipali Nale, and Roshani Agrawal ." Automatic text summarization." International Journal of Computer Applications, (2015):17.

[2] Abdel-fattah, Mahmoud A. "Arabic sign language: a perspective." Journal of Deaf Studies and Deaf Education (2005): 212-221.

[3] Ahmed Ibrahim, Tarek Elghazaly, Mervat Gheith." A Novel Arabic Text Summarization Model Based on Rhetorical Structure Theory and Vector Space Model ." International Journal of Computational Linguistics and Natural Language Processing, (2013):480-485.

[4] Alison Wray, Stephen Cox, Mike Lincoln,and Judy Tryggvason. "A formulaic approach to translation at the post office: reading the signs." Language \& Communication, (2004):59-75. 
International Journal of Information Technology, Control and Automation (IJITCA) Vol.7, No.3, July 2017

[5] Ameera Almasoud,and Hend Al-Khalifa."SemSignWriting: A Proposed Semantic System for Arabic Text-to-SignWriting Translation." JSEA, (2012) :604-612.

[6] Ani Nenkova. Kathleen McKeown. "Automatic summarization." Foundations and Trends® in Information Retrieval, (2011):103-233.

[7] Aqil Azmi, and Suha Al-Thanyyan. "A text summarizer for Arabic." Computer Speech \& Language, (2012):260-273.

[8] Basmah faraj, Wafa AL-rajhi, and Yahya elhadj." Avatar Based Approach for Teaching Arabic Sign Language." J. of Commun. \&Compute. Eng, (2012) : 43.

[9] Chang, Jae-Young ,KIM, and Il-Mi. " Research Trends on Graph-Based Text Mining." International Journal of Software Engineering and Its Applications, (2014):147-156.

[10] Daan Hermans ,Harry Knoors ,Ellen Ormel ,and Ludo Verhoeven. "The Relationship Between the Reading and Signing Skills of Deaf Children in Bilingual Education Programs." Journal of Deaf Studies and Deaf Education, (2008):518-530.

[11] David Inouye, Jugal K. Kalita."Comparing twitter summarization algorithms for multiple post summaries. "In Privacy, Security, Risk and Trust (PASSAT) and 2011 IEEE Third Inernational Conference on Social Computing (SocialCom), (2011):298-306 .

[12] Dipanjan Das, Andr_e F.T. Martins." A survey on automatic text summarization." Literature Survey for the Language and Statistics II course at CMU ,(2007): 192-195.

[13] Erkan, Radev ." LexPageRank: Prestige in Multi -Document Text Summarization ." In EMNLP, (2004) :365-371.

[14] Fatima AL-Khawaldeh,and Venus Samawi ."Lexical cohesion and entailment based segmentation for arabic text summarization (lceas). " The World of Computer Science and Information Technology Journal (WSCIT), (2015):51-60.

[15] Ganesan, K., Zhai, C., \& Han, J. . "Opinosis: a graph-based approach to abstractive summarization of highly redundant opinions." In Proceedings of the 23rd international conference on computational linguistics,(2010):340-348.

[16] Ghaleb Al_Gaphari, Fadl M. Ba-Alwi, and Aimen Moharram ."Text Summarization using Centrality Concept." International Journal of Computer Applications, (2013):5-12.

[17] Ghanem, S. and Albidewi, I. (2013)." An Avatar Based Natural Arabic Sign Language Generation System for Deaf People." Computer Engineering and Intelligent Systems, (2013):2222-1719.

[18] Hongyuan Zha."Generic summarization and keyphrase extraction using mutual reinforcement principle and sentence clustering." In Proceedings of the 25th annual international ACM SIGIR conference on Research and development in information retrieval ,(2002):113-120.

[19] Inderjeet Mani ."Summarization Evaluation: An Overview." In Proceedings of the NTCIR Workshop,(2001).

[20] Judith D. Schlesinger,Dianne P. O’Leary,John M. Conroy." Arabic/English Multi-document Summarization with CLASSY_The Past and the Future." International Conference on Intelligent Text Processing and Computational Linguistics, (2008):568-581.

[21] Martin Hassel. "Evaluation of automatic text summarization." Licentiate Thesis, Stockholm, Sweden, (2004):1-75. 
International Journal of Information Technology, Control and Automation (IJITCA) Vol.7, No.3, July 2017

[22] Marty Bray, David Pugalee, Claudia P. Flowers , and Bob Algozzine. "Accessibility of Middle Schools' Web Sites for Students with Disabilities." The Clearing House: A Journal of Educational Strategies, Issues and Ideas, (2007):169-178.

[23] Matjaž Debevc ,Primož Kosec ,Andreas Holzinger ." Improving multimodal web accessibility for deaf people: sign language interpreter module ." Multimedia Tools and Applications ,( 2011):181199.

[24] Mehrez Boulares, Mohamed Jemni." Toward an example-based machine translation from written text to ASL using virtual agent animation. "arXiv preprint (2012):1203.3023.

[25] MCLELlAN, Paulla A. "Web Accessibility". The College of St. Scholastica,(2011). PhD Thesis.

[26] Mine Berker. "Using genetic algorithms with lexical chains for automatic text summarization". Bogaziçi University,(2011). PhD Thesis.

[27] Mohamed Jemni ,Oussama Elghoul. "Towards Web-Based automatic interpretation of written text to Sign Language." In First International conference on ICT \& Accessibility, (2007).

[28] Mohammed Abo El-Soud , A.E. Hassan , M. S. Kandil , and Samaa M. Shohieb. "A Proposed Web Based Framework E-Learning and Dictionary System for Deaf Arab Students. " International Journal of Electrical \& Computer Sciences IJECS-IJENS, (2010):24-50.

[29] Mohandes." Automatic translation of Arabic text to Arabic sign language." AIML Journal, (2006): 15-19.

[30] N Dheer, C Kumar."Automatic Text Summarization: A Detailed Study. "International Journal of Science and Research . (2016): 429-433.

[31] Nabil Alami, Mohammed Meknassi, and Noureddine." Automatic texts summarization: Current state of the art. "Journal of Asian Scientific Research,( 2015):1.

[32] Nabil Hewahi,Kathrein Abu Kwaik ."Automatic Arabic Text Summarization System (AATSS) Based on Semantic Features Extraction. International Journal of Technology Diffusion, (2012):1227.

[33] Peter Turney."Learning algorithms for keyphrase extraction . " Information retrieval, (2000):303336.

[34] Qasem A . Al-Radaideh, and Mohammad Afif ."Arabic text summarization using aggregate similarity". In the international Arab conference on information Technology. (2011).

[35] Rada Mihalcea." Graph-based ranking algorithms for sentence extraction, applied to text summarization." In Proceedings of the ACL 2004 on Interactive poster and demonstration sessions ,(2004):20.

[36] Rafeeq Al-Hashemi . "Text Summarization Extraction System (TSES) Using Extracted Keywords . " Int. Arab J. e-Technol .(2012): 164-168.

[37] Rasim Alguliev , and Ramiz Aliguliyev . . Evolutionary algorithm for extractive text summarization." Intelligent Information Management (2009):128-138.

[38] Rucha S. Dixit, Prof. Dr.S.S.Apte. "Improvement of Text Summarization using Fuzzy Logic Based Method. "IOSRJCE, (2012):05-10.

[39] S.M. Halawani and Zaitun A.B. "An avatar based translation system from arabic speech to arabic sign language for deaf people." International Journal of Information Science and Education , (2012):13-20. 
International Journal of Information Technology, Control and Automation (IJITCA) Vol.7, No.3, July 2017

[40] Shashank gupta, Anushree Jagrawal, and Neha mathur. "Automatixc Text Summarization using Page Rank and Genetic Algorithim." Journal of Rajasthan Academy of Physical Sceiences, (2014):171-179.

[41] Sobh, Darwish. and Fayek. . "Evaluation Approaches for an Arabic Extractive Generic Text Summarization System." In: proceeding of 2nd International Conference on Arabic Language Resource and Tools. (2009):150-155.

[42] Thomas Hanke. "HamNoSys-representing sign language data in language resources and language processing contexts." In LREC, (2004).

[43] Waleed AlSanie, Touir, and Mathkour. "Towards an infrastructure for Arabic text summarization using rhetorical structure theory". Master's thesis, King Saud University, Riyadh.(2005):2-70.

[44] Yihong Gong, Xin Liu." Generic text summarization using relevance measure and latent semantic analysis." In Proceedings of the 24th annual international ACM SIGIR conference on Research and development in information retrieval, (2001) 19-25.

[45] Yosra Bouzid, Mohamed Jemni. "An Avatar based approach for automatically interpreting a sign language notation." In: Advanced Learning Technologies (ICALT), 2013 IEEE 13th International Conference on. IEEE, (2013) :92-94.

[46] Yang, H., Liao, W. and Long Lay, Y. "Text to Sign Language Conversion." ,(2001)":241-249.

\section{Authors}

Ahmad A. Alhamed ,Ph.D. Assistant Professor Information Systems Department. College of Computer and Information Sciences. King Saud Universtiy,Riyadh,Saudi Arabia . aalhamed@ksu.edu.sa

Maha Saas Alqhtani, Teacher Assistance, Information Systems Department. College of Computer and Information Sciences.King Khalid Universtiy,Abha,Saudi Arabia . 